Check for updates

Cite this: RSC Adv., 2017, 7, 19924

\title{
Stabilizing silica nanoparticles in hydrogels: impact on storage and polydispersity $\dagger$
}

\author{
Giorgia Giovannini, ${ }^{a}$ Filip Kunc, ${ }^{a}$ Carmen C. Piras, ${ }^{a}$ Ondrej Stranik, ${ }^{b}$ Alison A. Edwards, ${ }^{a}$ \\ Andrew J. Hall ${ }^{a}$ and Vladimir Gubala (D) *a
}

For successful nanomedicine, it is important that the unique, size-dependent physico-chemical properties of the nanomaterial remain predictably constant during both the storage and the manipulation of the material. Here a novel approach to preserve the colloidal stability and degradation of NPs is described. The concept is simple: (a) a solution of monodisperse particles is formulated into a responsive water- or PBS-based hydrogel; (b) the gel can be reversibly turned into a solution after long term storage by shaking it by hand; (c) the NP can be diluted and used in any desired application without the need for excessive manipulation. The differences between the physico-chemical properties of NPs stored in solution and in gel are compared. Two types of NPs were involved in this study: silica NPs of $\sim 100 \mathrm{~nm}$ and Au-NPs of 30 and $80 \mathrm{~nm}$ in diameter. The key findings are: the fibrous matrix of the hydrogel limits the NP mobility, significantly reduces NP aggregation and conserves the NP morphology; both the hydrogelator and the NPs show negligible toxicity towards the model U937 human hematopoietic cell line; undesired leaching of cargo material loaded inside the particles is reduced, which could be an important feature for drug delivery systems.

Received 27th February 2017 Accepted 27th March 2017

DOI: 10.1039/c7ra02427d

rsc.li/rsc-advances culture media diminishes the effects of the electrostatic repulsions and increases the probability of aggregation. Number of theoretical (e.g. the DLVO theory ${ }^{7,8}$ ) and experimental approaches to stabilise NPs in liquid media have been proposed. Kamiya and Iijima comprehensively reviewed range of post-synthesis and in situ surface modification techniques for dispersion of nanoparticles in various solvents. ${ }^{9}$ For example, Sapra et al. improved water-dispersed nanoparticle suspension for at least 30 days by surface modification of nanoparticles during aerosol or gas-phase synthesis, followed by direct transfer into liquid media. ${ }^{10}$ And of course significant amount of research has been done to stabilise silica (SiNP) and gold (AuNP) ${ }^{11}$ nanoparticles, two good examples of widely used nanomaterial in biological applications. For AuNPs, the most common stabilisation methods rely on the use of ionic and nonionic surfactants. ${ }^{12,13}$ For SiNPs, one of the most elegant solution was proposed by Bagwe. ${ }^{5}$ It relies on electrostatic repulsions between NPs, which is done by simple, post-synthetic introduction of organosilanes with charged and chemically "inert" functional groups on the SiNP surface. SiNP also attracted much attention because it is possible to incorporate various hydrophobic and hydrophilic cargo inside the silica matrix,${ }^{14}$ which made them popular material to be studied as drug delivery systems (DDS)..$^{15}$ It is easy to store nonfunctionalised SiNPs in organic solvent such as ethanol for long term, however functionalized SiNP (e.g. equipped with antibodies or sensitive, biologically active cargo) cannot be stored in organic solvents and are known to aggregate in
${ }^{a}$ Medway School of Pharmacy, University of Kent, Central Avenue, Chatham, ME44TB, UK. E-mail:V.Gubala@kent.ac.uk

${ }^{b}$ The Leibniz Institute of Photonic Technology (IPHT), Albert-Einstein-Straße 9, 07745 Jena, Germany

$\dagger$ Electronic supplementary information (ESI) available. See DOI: $10.1039 / \mathrm{c} 7 \mathrm{ra} 02427 \mathrm{~d}$ 
enriched aqueous solutions such as buffers, ${ }^{\mathbf{1 6}}$ cell culture media or serum. ${ }^{17}$ Once nanoparticles aggregate in solution, the most common manipulation to redisperse the sample involves the use of ultrasound to 'break' the aggregates into a monodispersed suspension. However, access to ultrasonic bath or probe is not always possible and some of the mechanical energy used to move and vibrate NPs in solution could also be transferred to another undesired processes associated with a decomposition of the material, premature leaching of the drug load, denaturation of proteins (if used) or in case of SiNPs to the acceleration of a process called Ostwald ripening. ${ }^{18-21}$ Therefore, a new method for formulation and storage of NPs would be welcome. Ideally, the alternative method should be straightforward, and extremely simple for the end user to do. The requirement for simplicity of the NP-sample manipulation stems from the fact that the end user often isn't the material chemist that prepared the material but someone from quite different scientific discipline (e.g. biological and environmental sciences, medicine etc.). In our previous work, we have explored the possibility of formulating silica nanoparticles (NPs) in

a)
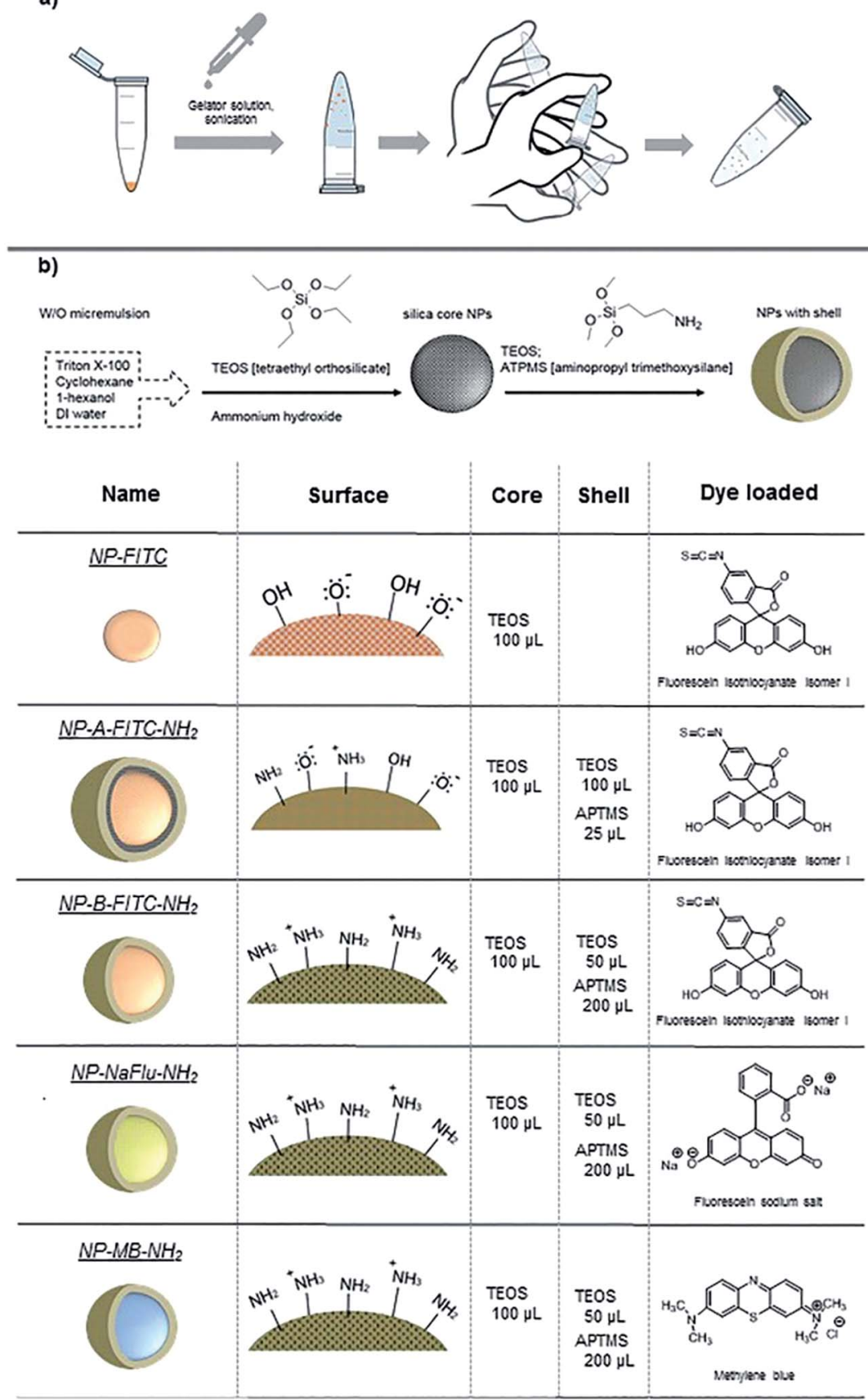

Fig. 1 (a) Illustration of the general concept, (b) scheme showing the synthesis of SiNP; the types and specifications of nanomaterial studied in this work. 
freeze-dried solid forms upon addition of cryoprotectants. ${ }^{22}$ While this method was relatively simple, it was also time consuming and limited to material that could tolerate freezing and dehydration stress. Here we report on a new, more generic approach by formulating silica nanoparticles into responsive hydrogels.

The combination of hydrogels and nanoparticles to enhance the properties of the individual components for various applications has been well studied. The concepts, design and applications of nanoparticle-hydrogel composites were comprehensively reviewed by Thoniyot et $a .^{23}$ Stimuli-responsive AuNP-gel and SiNP-gel hybrids showed promising efficacy in surface plasmon resonance (SPR) based sensors, ${ }^{24}$ anti-bacterial applications, ${ }^{25}$ as medicated dressings to prevent infection in chronic wounds, ${ }^{26}$ in thermoreversible ${ }^{27}$ and temperature sensitive ${ }^{28}$ hydrogels for drug delivery, ${ }^{29}$ for improved tissue adhesion, ${ }^{30}$ in electrochemical applications and optoelectronic devices ${ }^{31}$ among others. However, simple approach of a well-designed, mechanically responsive hydrogel that would be a great alternative for the standard, liquid formulation of nanoparticles, while preserving their colloidal stability is still missing.

In this work, we trapped nanoparticles in a reversible hydrogel prepared from low-molecular-weight gelator and studied the effect of this formulation on their colloidal stability. To demonstrate that this method was not exclusive only to preserve the colloidal stability of SiNPs, we have also used commercially available AuNPs (30 and $80 \mathrm{~nm}$ in diameter). The concept is illustrated in Fig. 1a. The critical prerequisites of our new vehicle for nanoparticle storage were: (1) the gelator must be inert to the nanomaterial; (2) nanoparticles and gel must be easily separable without any chemical alteration; (3) the hydrogel must be able to withstand certain level of mechanical stress but also be able to turn back into monodispersed suspension with minimum user manipulation (e.g. by shaking the sample by hand) and (4) once the hydrogel is turned back to solution, any remaining gel-fibre debris must be biocompatible and non-toxic. Considering these conditions, an amphiphilic molecule, Fmoc-galactosamine (Fmoc-gal) was chosen as the best candidate to form a responsive hydrogel when added to the NP suspension. This derivative is well-studied, chemically inert when in contact with nanoparticles and its various structural analogues were already explored for instance in the preparation of cell culture scaffolds. ${ }^{32}$ The mechanically responsive hydrogel was generated by the self-assembly of Fmoc-gal (at $2 \mathrm{mg} \mathrm{mL}^{-1}$ ), which was mixed with the aqueous NP suspension. We have tested this concept on several types of in-house made SiNPs and commercially sourced $30 \mathrm{~nm}$ and $80 \mathrm{~nm}$ gold nanoparticles.

For SiNPs, we have compared the longer term colloidal stability of dye doped silica nanoparticles prepared by microemulsion method both with net negative charge and with net positive charge suspended in water, phosphate buffer saline (PBS) and also when formulated in a supramolecular hydrogel. The specifics of the SiNPs used in this study are summarized in Fig. 1b. Because SiNPs are good models for DDS, we studied the SiNP ability to retain their hydrophobic and hydrophilic cargo while being stored over longer period of time in the gel. In a model experiment, we have compared the effect of the dye- leaching phenomenon (i.e. analogous to controlled drug release) between three formulations: (i) NPs in organic suspension, (ii) in aqueous suspension and (iii) in the hydrogel. Three different dyes (as drug surrogates) with ranging hydrophobicity - hydrophilicity were incorporated inside the NP core to provide insights into the stability of the silica matrix when formulated into the gel. Some of the engineered nanomaterial, especially the ones intended to be used in medicine will be equipped with often light-sensitive drugs, organic/inorganic dyes or functionalised on their surface with biorelevant material such as antibodies or oligonucleotides. For such reasons, the NPs in this study were stored in the dark, at $4{ }^{\circ} \mathrm{C}$. The analyses were performed at room temperature and the model drug release experiment was conducted at $37^{\circ} \mathrm{C}$ to mimic the physiological temperature.

\section{Results and discussion}

\section{NP preparation and formulation}

While the AuNPs used in this study were obtained from commercial sources, the SiNPs were prepared in-house by reverse microemulsion method. ${ }^{33}$ The size and surface charge of the prepared SiNPs was measured by dynamic light scattering both in water and ethanol. The size, the monodispersity and the fact that the SiNPs were of spherical shape was then corroborated by transmission electron microscope as illustrated in Fig. 2. As a reference, we have firstly prepared plain fluorescein isothiocyanate-doped silica NPs (NP-FITC), which exhibit surface negative charge due to the presence of silanols. The FITC molecules were covalently incorporated inside the silica matrix using aminated organosilane (APTMS) as a cross-linker, following a method described by Tan et al. previously. ${ }^{33}$ Summarized in Table 1, the plain SiNPs had a diameter of 60 $\mathrm{nm} / 72 \mathrm{~nm}$ and zeta potential of $-30 /-34.4 \mathrm{mV}$ in ethanol and water respectively.

The size of the individual SiNPs measured by TEM was $38 \mathrm{~nm}$. The discrepancy between the two techniques were attributed to the fact that the TEM images were acquired in vacuum from SiNPs dried on the copper grid. In an attempt to prepare SiNPs with less negative surface charge, we synthesized FITC-doped silica NPs with an outer shell composed of combination of the negatively charged silanols and limited amount of positively charged amines (TEOS : APTMS $=4: 1$ by volume). This type of SiNPs were labelled as NP-A-FITC-NH$H_{2}$.

The thick layer of TEOS grafted on the SiNP surface caused the NP-A-FITC- $\mathrm{NH}_{2}$ grow to diameter of $157 \mathrm{~nm} / 101 \mathrm{~nm}$ with surface charge of $-18 /-22.5 \mathrm{mV}$ in ethanol and water respectively (Table 1). Interestingly, the presence of the shell resulted in NPs with much brighter yellow/orange colour when compared to the NP-FITC without the shell (Fig. 2, right column). This visible 'brightness' increase is presumably attributed to entrapping FITC molecules in the different environments of the SiNPs with and without the shell. The final three types of SiNPs involved in this study were synthesized with identical surface chemistry, the only difference was the dye used. Fluorescein isothiocyanate (FITC), highly water soluble sodium salt of fluorescein (NaFlu) and water insoluble 


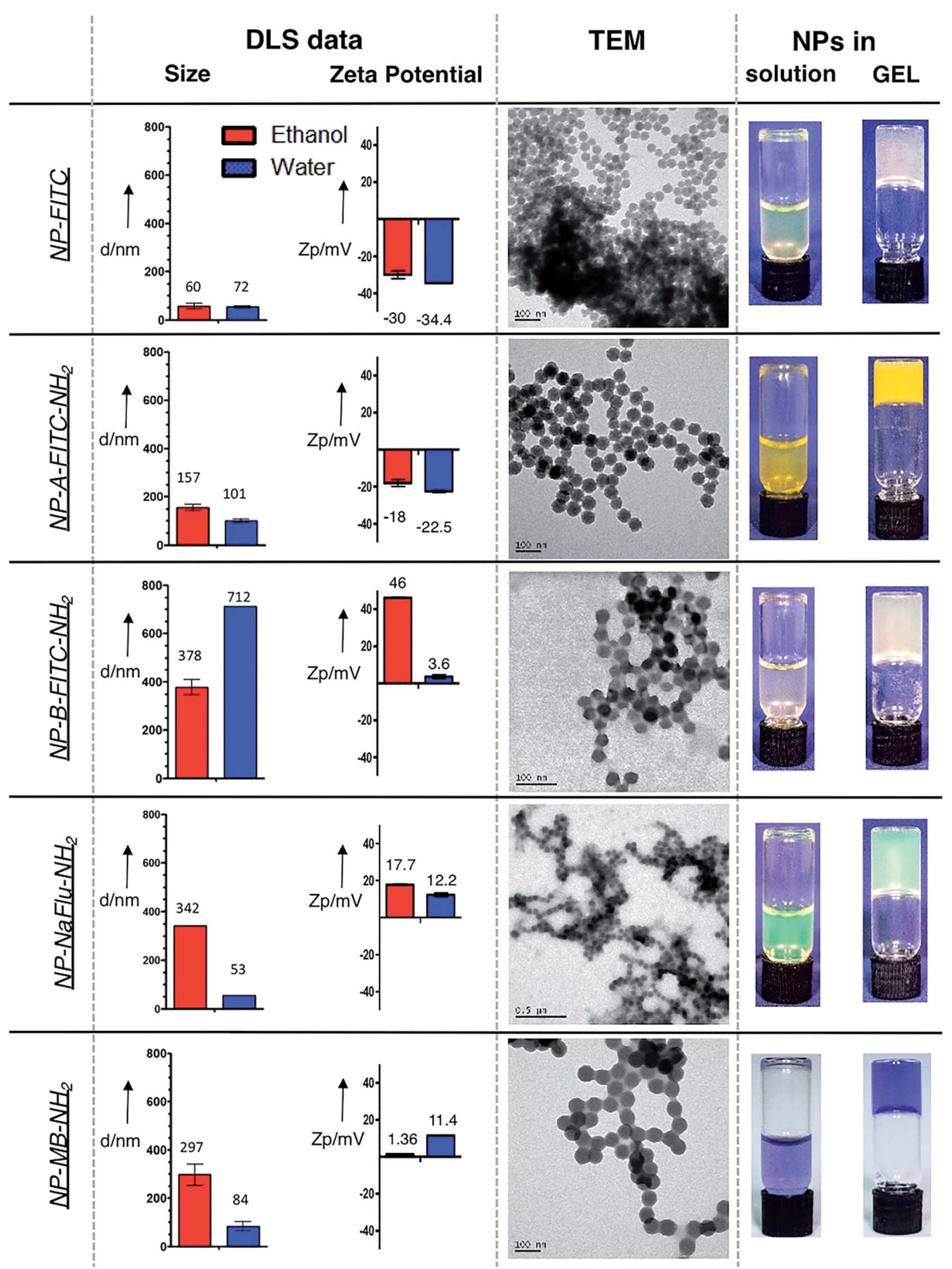

Fig. 2 DLS and TEM data showing the size, charge and the morphology of the prepared SiNPs. Right column shows the SiNP suspension and the SiNPs in gel.

methylene blue (MB) were doped inside SiNPs (Fig. 1) and modified on the surface with relatively thick shell consisting of polymerized TEOS : APTMS molecules in a ratio of $1: 4$ (by volume). Positively charged, APTMS-coated silica nanoparticles (with higher density of $-\mathrm{NH}_{2} /-\mathrm{NH}_{3}{ }^{+}$groups) are known to aggregate readily in liquids, which created a great opportunity for us to test the ability of the hydrogel to stabilize the colloidally 'highly unstable' SiNPs.
The fact that the positively charged APTMS-coated SiNPs tend to aggregate in solution is evident from Fig. 2. Although the TEM pictures show that the size of the individual SiNPs is still in the range of $62-89 \mathrm{~nm}$, DLS data indicate significant aggregation with average size in the range of $297-378 \mathrm{~nm}$ in ethanol and even larger range in water. Importantly, as shown in Fig. 2, we were able to formulate each prepared SiNP sample into a suspension and also into a gel. 
Table 1 Diameter, polydispersity index (PDI) and zeta potential of NPs measured by DLS in ethanol and water and by TEM in vacuum

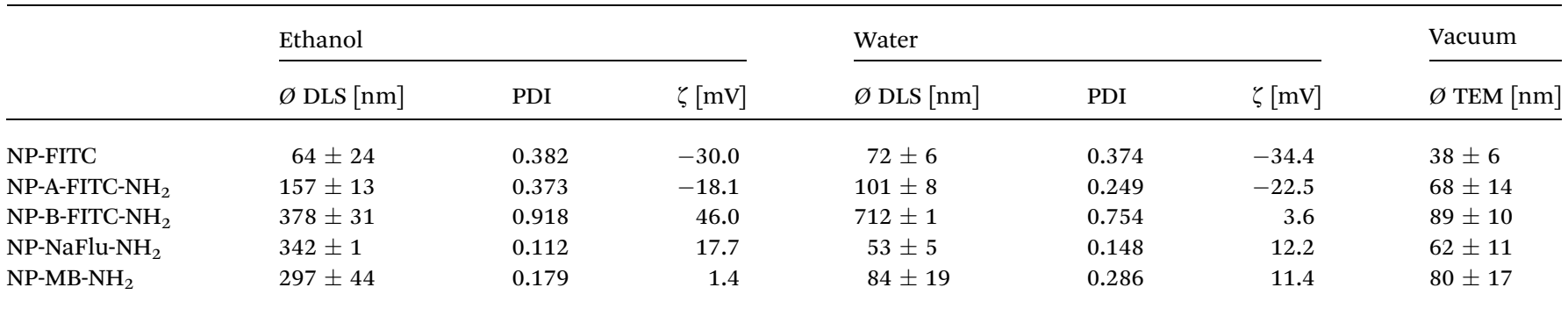

\section{Colloidal stability - long term storage}

The in-house prepared SiNP and two commercially sourced AuNPs samples were formulated into an aqueous suspension (in water) and hydrogels at the concentration of $1 \mathrm{mg}$ of NP per $\mathrm{mL}$, which corresponds to approximately $\sim 1 \times 10^{12}$ NPs per $\mathrm{mL}$. The concentration of the Fmoc-gal gelator was $2 \mathrm{mg} \mathrm{mL}^{-1}$. Both the aqueous and hydrogel formulations were stored at $4{ }^{\circ} \mathrm{C}$ for either several days or weeks. After storing the NPs for set amount of weeks, both the aqueous and hydrogel NP samples were gently shaken by hand for the hydrogel to convert back to liquid and then diluted to $0.5 \mathrm{mg}$ of NP per mL. This represents an optimal concentration required for accurate analysis of the size and polydispersity by Dynamic Light Scattering (DLS), Nanoparticle Tracking Analysis (NTA) and Transmission Electron Microscopy (TEM). Importantly, we have previously reported on the use of silica NPs as drug delivery carriers and demonstrated that the concentration of NPs for in vitro and in vivo experiments is optimal in the range of $1-0.001 \mathrm{mg}$ of NP per mL. ${ }^{18}$ Qualitatively, as seen on Fig. S1 and S2, $\dagger$ it was possible to observe the aggregation of both AuNPs and SiNPs stored in a solution by a naked eye as early as after 1 week of storage. Storing AuNPs in solution for one week resulted in significant aggregation, evident by the high polydispersity and particle average size of $\sim 175 \mathrm{~nm}$. On the other hand, the AuNPs stored in the hydrogel formulations appeared to have no visible aggregates. Quantitative analysis of the particle size inside the hydrogel or when the AuNPs were dispersed to suspension by 'breaking' the gels proved to be more challenging due to the presence of gel fibre debris. Therefore, the DLS data were corroborated by NTA, a technique that is not affected by the presence of gel fibre debris. The analysis of NTA data, shown on Fig. S3, $\dagger$ indicate that the size distribution of AuNPs stored in hydrogel is significantly improved when compared to the AuNPs stored in solution.

SiNPs have also high tendency to aggregate in solution, however in this case the silica matrix is also prone to hydrolysis, which leads to dissolution of the SiNPs. In order to study the colloidal stability of SiNPs and their changes in morphology, we stored SiNPs both in solution and hydrogel for 30 days and used TEM for analysis (Fig. 3). TEM provided clearer picture of the various SiNP morphologies, the distinct populations present in the mixture and the presence of the gel-fibre debris was not detrimental to the analysis and interpretation.

As seen on Fig. 3 (with Gaussian distribution on Fig. S4†), when all five SiNP samples were synthesized and placed in

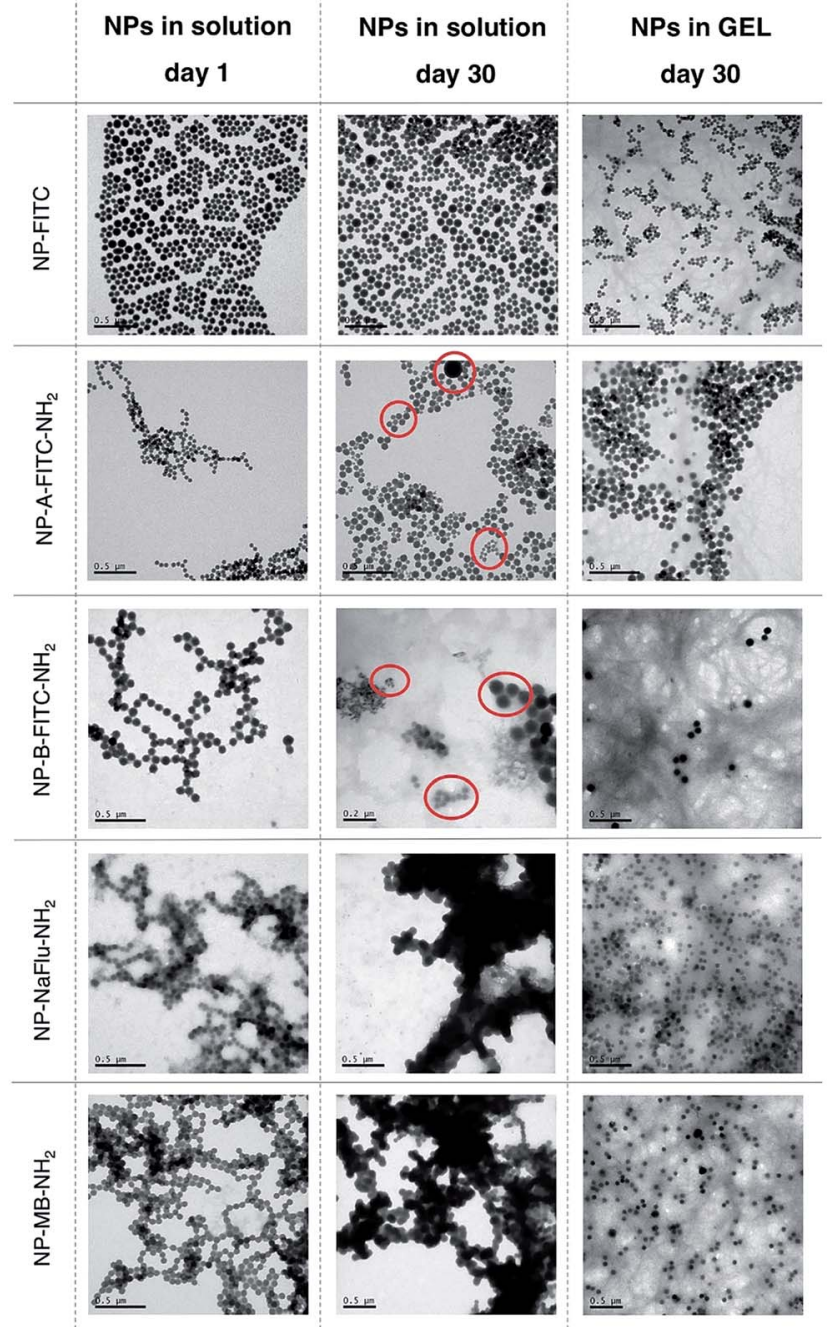

Fig. 3 TEM images showing the evolution of the SiNP morphologies and size distribution when stored in water for 1 day and 30 days (left and middle column) and in gel for 30 days (right column). The red circles highlight the evolution of various SiNP populations over time, which clearly demonstrates that the silica NPs are behaving as a dynamic system in water. On the other hand, when stored for 30 days in gel, the morphology and uniformity of the SiNPs is extremely monodispersed. The $0.2 \mu \mathrm{m}$ scale for NP-B-FITC- $\mathrm{NH}_{2}$ was used on purpose to illustrate the various NP populations. Storing NP-NaFlu$\mathrm{NH}_{2}$ and NP-MB-NH in solution for 30 days resulted in significant NPpolymerisation/aggregation, which means that this material should no longer be classified as nanomaterial. 
water, they were of uniform size with relatively narrow size distribution. No visible aggregation was observed, although DLS data (Table 1) suggest some degree of NP-NP interactions for the positively charged SiNPs (NP-B-FITC-NH ${ }_{2}, \mathrm{NP}-\mathrm{NaFlu}-\mathrm{NH}_{2}$ and NP-MB-NH $\mathrm{N}_{2}$ ). After storing the SiNPs in solution for 30 days, clear indication of polydispersity was evident across all tested samples. In case of NP-A-FITC-NH $\mathrm{N}_{2}$ and NP-B-FITC-NH $\mathrm{N}_{2}$, two or even three very distinct populations of SiNPs were observed (Fig. 3, red circles). Apart from the original $\sim 75 \mathrm{~nm}$ particles, we have observed a population of some ultrasmall, $\sim 20 \mathrm{~nm}$ spherical SiNPs and also some very large, $\sim 200 \mathrm{~nm}$ species.

This phenomenon could be explained by a process known as Ostwald ripening, in which monomers dissolved from smaller silica particles contribute to the growth of larger ones and the particle size distribution constantly evolves towards an equilibrium. ${ }^{19}$ This process is mainly observed during the formation of particles when the catalyst is present, however it unavoidably continues when SiNPs are stored in solutions too, since silica condensation is reversible. ${ }^{20}$ Importantly, the evolution of various populations of silica NPs is not observed in the hydrogel samples. We assume that the mobility of SiNPs stored in gel is inhibited by the gel fibers, hence both the dissolution of silica matrix and Ostwald ripening are retarded. A net results of storing SiNPs in such less dynamic system is that the structural integrity of SiNPs and their content can be secured for longer period of time. NP-NaFlu- $\mathrm{NH}_{2}$ and NP-MB- $\mathrm{NH}_{2}$ samples were highly aggregated when stored in solution for 30 days, with visible precipitates suggesting a very unstable colloidal system (Fig. 3, middle column). In fact, the precipitates were clearly visible in the solution by a naked eye even after 1 week of storage (Fig. S1†).

These findings were in stark contrast with the images obtained from the hydrogel samples. Although the debris from the gel fibres were still visible under the TEM, the SiNPs were highly monodispersed, with retained shape and extremely narrow size distribution. We have observed no deformation of the SiNPs, no evidence of extensive hydrolysis, no nanoparticle-bridging and no visible precipitates. The forces that usually govern the motion and aggregation of the particles in solution were restricted inside the hydrogel, which translated to a highly stable SiNP sample after storing it for 30 days.

\section{Dye leaching - silica degradation}

A critical feature for any successful drug delivery systems (DDS) is the ability to undergo biodegradation (or self-degradation) but also to retain the clinically relevant concentration of the drug while in storage. Firstly, we have studied the capacity of NP-B-FITC-NH $\mathrm{N}_{2}, \mathrm{NP}-\mathrm{NaFlu}-\mathrm{NH}_{2}$ and NP-MB- $\mathrm{NH}_{2}$ to retain their cargo when stored in ethanol and water for 3 weeks. The amount of released dye from the silica NPs was tracked by measuring the fluorescence of the SiNPs and the supernatant at different time intervals while keeping the samples in suspension for 21 days (Fig. S5†). The differences in dye-leaching between the studied solvents were quite remarkable. All three studied dyes were leaching relatively slowly when the SiNPs were stored in EtOH but the rate of leaching was significantly accelerated in water. While the water-soluble salt of fluorescein in NP-NaFlu- $\mathrm{NH}_{2}$ is only physically adsorbed inside the silica pores, the FITC dyes in NP-B-FITC- $\mathrm{NH}_{2}$ are covalently incorporated into the silica matrix via organosilane linkage. However, because the leaching rate of both dyes (covalently and noncovalently linked to silica matrix) from the SiNPs in aqueous environment is equally fast, this suggests rapid degradation of the silica matrix in water. Although this might be beneficial for drug delivery as it satisfied the requirement of degradability of the nanomaterial, it represents a challenge for the storage of silica based DDS.

To gain more insight on how fast silica degradation could be under a model physiological conditions, we have monitored the dye leaching of NP-B-FITC-NH $\mathrm{N}_{2}, \mathrm{NP}-\mathrm{NaFlu}-\mathrm{NH}_{2}$ and NP-MB-NH when shaken in an isotonic medium (0.01 M PBS pH 7.4 with

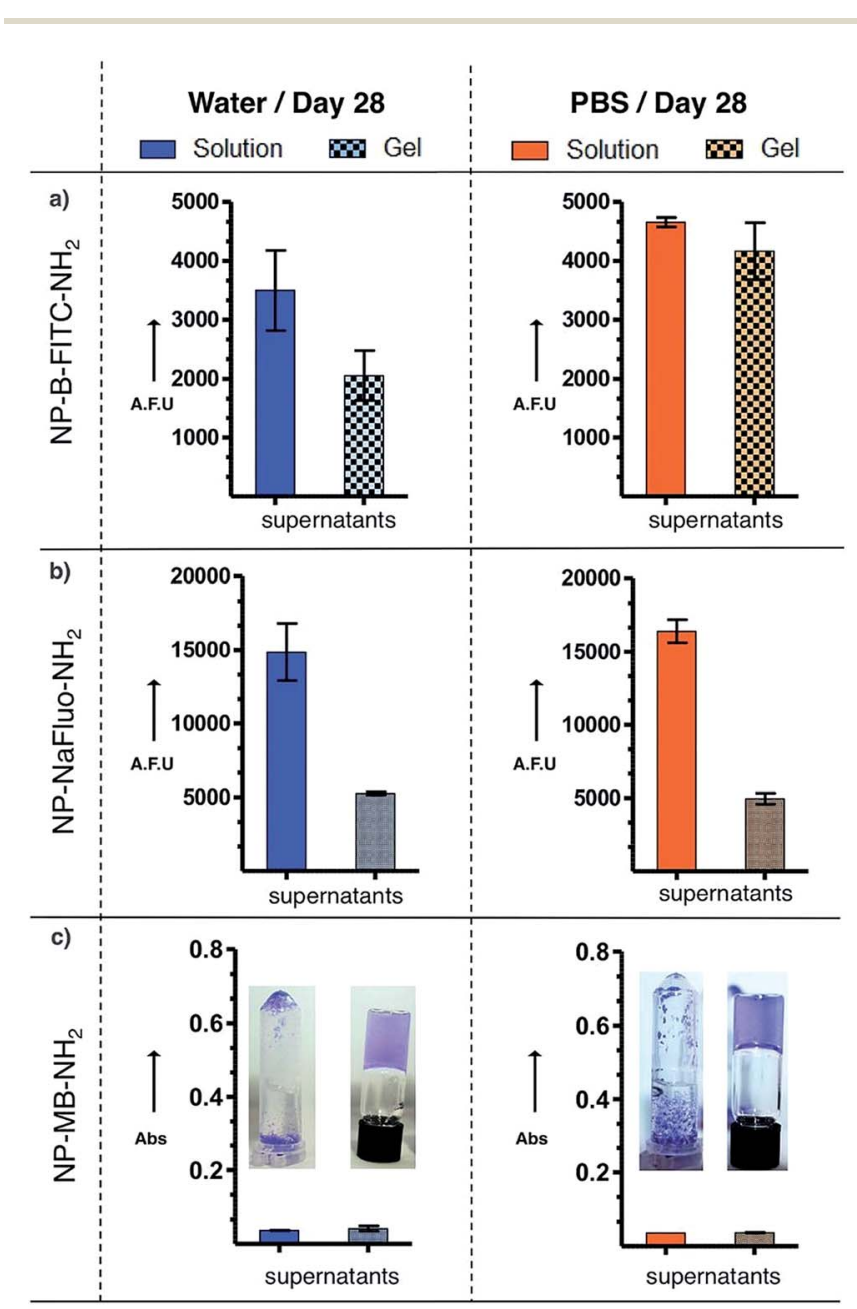

Fig. 4 Leaching of the dye from SiNPs to the supernatant while being stored in solution or in gel. (a) FITC covalently linked to silica matrix the leaching was partially reduced when stored in gel (excitation/ emission wavelengths: $471 / 525 \mathrm{~nm}$ ). (b) FITC-sodium salt physically trapped in the silica matrix - the amount of dye leached from the NPs is dramatically reduced when stored in gel (excitation/emission wavelengths: 456/518 nm). (c) Methylene blue physically trapped in the silica matrix - very slow leaching of the hydrophobic dye in both formulations but very dramatic difference in colloidal stability of the NP-B-FITC-NH $\mathrm{N}_{2}$ when stored in water and gel (absorbance: $664 \mathrm{~nm}$ ). 
$0.137 \mathrm{M} \mathrm{NaCl}$ ) at $37{ }^{\circ} \mathrm{C}$ for 8 hours. For reference purposes, we have performed identical experiments in ethanol and water. The results are summarized in Fig. S6. $\dagger$ The dye release, as a consequence of silica matrix hydrolysis was very prominent both in water and PBS in the case of NP-B-FITC- $\mathrm{NH}_{2}$ and NPNaFlu- $\mathrm{NH}_{2}$. Significant accumulation of FITC and the watersoluble fluorescein analogue $\left(\mathrm{Na}^{+} \mathrm{Flu}\right)$ in the supernatant was evident immediately after several minutes. On the other hand, methylene blue, as the most hydrophobic dye was not released into the aqueous environment readily. In fact, the release of methylene blue from NP-MB- $\mathrm{NH}_{2}$ was slow in both water and ethanol and only a small degree of accelerated release of methylene blue was observed in PBS after 6 hours. These simple experiments highlighted two important requirements: (1) it is preferable to store silica NPs at $4{ }^{\circ} \mathrm{C}$ and (2) it is necessary to formulate the silica NPs into a preparation, where the dye leaching effect could be minimized.

\section{Dye leaching - long term storage}

To demonstrate the advantage of the hydrogel approach, NP-BFITC-NH ${ }_{2}, \mathrm{NP}-\mathrm{NaFlu}-\mathrm{NH}_{2}$ and NP-MB- $\mathrm{NH}_{2}$ were stored for 28 days at $4{ }^{\circ} \mathrm{C}$ in water-based Fmoc-Gal hydrogel and also PBSbased Fmoc-Gal hydrogel. On the 28th day, all samples were gently shaken by hand, the resulting suspension was diluted to $0.5 \mathrm{mg}$ of SiNP per $\mathrm{mL}$, centrifuged to separate the supernatant, which was analysed by fluorescence (absorbance in the case of methylene blue). As a reference, identical procedure was followed with SiNPs stored in water and PBS in the absence of the gelator. The results are presented in Fig. 4 and S7. $\dagger$

The hydrogel had stabilizing effect on both the dye leaching and the colloidal stability of the measured NPs. The most notable differences between the NPs' capacity to retain their cargo when stored in solution and gel was observed in case of
NP-NaFlu- $\mathrm{NH}_{2}$. The degradation of the SiNPs and the subsequent release of the dye was three times slower in the gel (for both water-based gel and PBS-based gel) when compared to the corresponding solutions. Less dramatic effect was observed in the case of NP-B-FITC- $\mathrm{NH}_{2}$. Remarkably, despite that the hydrophobic methylene blue leached out of the NPs very slowly in both formulations, the differences in the colloidal stability of NP-MB- $\mathrm{NH}_{2}$ between water and gel were enormous (Fig. 4C).

\section{NP-hydrogel stability}

So far it was established that the prepared hydrogel is very stable while being stored 'still' (i.e. without shaking or strong vibrations) for long time and that it is possible to 'break' it into a solution by simply shaking it in hand. However, we recognized the need to test the ability of this new formulation to retain the protective, gel-like properties as a function of time and external vibrations, and how this ability correlates with some intrinsic factors of the SiNPs such as charge and the nature of the cargo inside the NPs. To do this, all SiNPs were formulated into gels and exposed to strong vibrations using a simple orbital shaker. The conversion of the hydrogel into a solution as a function of shaking speed and time was then qualitatively compared.

Some interesting trends were observed (Fig. S8-S12†). Firstly, the gel stability positively correlated with increased zeta potential. Secondly, the more hydrophobic the dye was, the more stable the gel appeared to be. The first trend is clearly showed on Fig. 5. The NP-A-FITC- $\mathrm{NH}_{2}(\zeta=-22.5 \mathrm{mV})$ were evidently converted from the gel to a suspension after 29 hours and 30 minutes of shaking at $1200 \mathrm{rpm}$, while the NP-B-FITC$\mathrm{NH}_{2}(\zeta=+3.6 \mathrm{mV})$ retained its self-supporting gel characteristic even after and 30 hours of shaking at $1200 \mathrm{rpm}$. Similarly, the highly negatively charged NP-FITC $(\zeta=-34.4 \mathrm{mV})$ were found to convert to a suspension at $800 \mathrm{rpm}$ and after more than 7

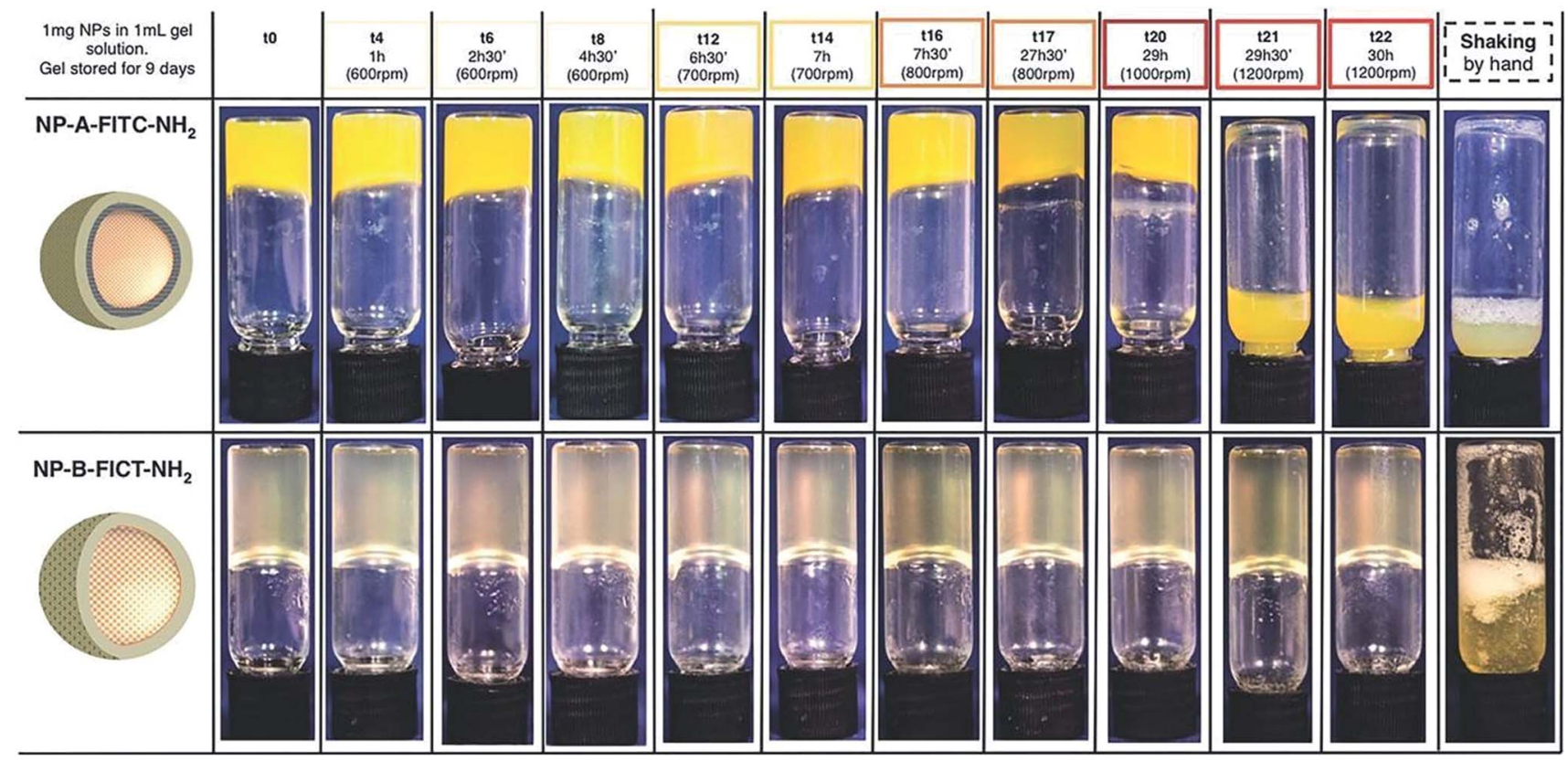

Fig. $5 \mathrm{NP}-\mathrm{A}-\mathrm{FITC}-\mathrm{NH}_{2}$ and NP-A-FITC-NH gel formulations shaken by orbital shaker for up to 30 hours with increasing speed. 
hours of shaking. The second trend is best illustrated in Fig. S8 and $59 . \dagger$ The NP-MB- $\mathrm{NH}_{2}$ with the hydrophobic methylene blue had very positive effect on the gel stability, while the NP-NaFlu$\mathrm{NH}_{2}$ with the highly soluble NaFlu dye had detrimental effect on the integrity of the gel. Presumably NPs with highly water soluble cargo will need to be stored under conditions avoiding strong vibrations or they will require to be formulated in a gel with higher stiffness than the one reported here.

\section{NP-hydrogel in vitro cytotoxicity}

Silica nanoparticles have been studied in several biomedical applications, both in vitro and in vivo and their low cytotoxicity is relatively well documented ${ }^{\mathbf{1 8 2 1 , 2 2}}$ Once formulated in a hydrogel however, even basic toxicology data are not available. The typical concentration of silica NPs used either in animal studies or in vitro work ranges between 1-0.001 $\mathrm{mg}$ of NP per mL. In this work, we have observed that once the NP-gel formulation was converted back to suspension and diluted to $0.5 \mathrm{mg}$ of NP per $\mathrm{mL}$, some of the hydrogel fibres remained in the suspension and they were clearly visible under TEM (Fig. 3). Although further dilutions could dissolve the gel fibres completely, the cytotoxic effect of either the diluted Fmoc-Gal or some of the remaining nano-sized gel fibres needed to be investigated. This was done by performing an MTS Cell Proliferation Assay on human glioblastoma astrocytoma cell line (U87MG). This assay allow to evaluate the cell viability determining their metabolic activity. If cells are alive and metabolically active, they produce mitochondrial dehydrogenase. This enzyme reduces MTS's reagent (tetrazolium salt), forming a product that absorbs light at $490 \mathrm{~nm}$. U87MG cells have been chosen because they adhere well on surfaces. This property allows to remove the analysed sample after incubation with cell, limiting interferences between the sample and MTS's reagent. To obtain basic cytotoxicity data, three formulations were studied: SiNP in hydrogel, SiNP in solution and Fmoc-Gal hydrogel without SiNPs. All formulations were allowed to incubate with U87MG cells for 1, 2 and 4 hours in a 96-well plate format in a cell culture medium. The assay has been performed in triplicate and the results are shown in Fig. 6. The cytotoxic effect either of the gel or the silica nanoparticles was minimal. In fact, the

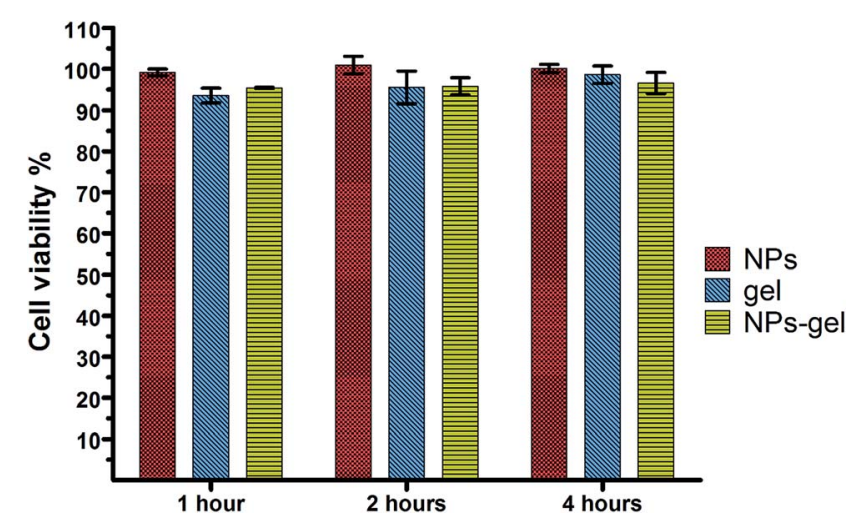

Fig. 6 Cell viability measured by MTS assay with U937 human hematopoietic cell line incubated with silica nanoparticles (red bars), hydrogel (blue bars) and SiNPs in hydrogel (green lines). proportion of live cells was marginally increasing over time, suggesting that the interaction of Fmoc-Gal, SiNPs or both with U87MG cells did not have detrimental effect on the cell life. Additional in vivo nanotoxicology studies are currently being conducted to corroborate these findings.

\section{Conclusion}

In summary, we have presented a simple and perhaps the most universal solution to storing silica NPs without significant effects to their colloidal stability and degradation. The elegant hydrogel restricts the movement of the individual nanoparticles and slows down any undesired ongoing reactions of the silica matrix or collisions of particles. This in turns presents obvious benefits in terms of nanoparticle aggregation and drug release. An initial cytotoxicity data showed negligible effect on cell growth, however additional in vivo toxicology tests are currently in progress in our laboratory to fully elucidate the impact of these findings on live biological systems. Because of its simplicity and versatility, our method has the real potential to be exploited by scientists working with various other types of NPs. We're confident that our findings could be very significant especially for the material scientists active in the field of nanomedicine. We hope that this could finally accelerate the progress towards the development of real, commercially available products that will revolutionize healthcare.

\section{Experimental section}

\section{Materials}

Cyclohexane (anhydrous, 99.5\%), 1-hexanol (anhydrous, $\geq 99 \%$ ), Triton ${ }^{\circledR}$ X-100, aminopropyl trimethoxysilane [APTMS] (97\%), tetraethyl orthosilicate [TEOS] (99.99\%), ammonium hydroxide solution $(28 \% \mathrm{w} / \mathrm{v}$ in water, $\geq 99.99 \%)$, fluorescein isothiocyanate isomer I, fluorescein sodium salt, sodium pyruvate solution. All mentioned were purchased from Sigma Aldrich. Methylene blue was purchased from Acros Organics. DMEM, antibiotic-antimycotic (containing 10000 units per $\mathrm{mL}$ of penicillin, $10000 \mu \mathrm{g} \mathrm{mL}^{-1}$ of streptomycin and $25 \mu \mathrm{g} \mathrm{mL} \mathrm{m}^{-1}$ of FungizoneTM) and fetal bovine serum (FBS) were purchased from GIBCO (Life Technologies). The model cell line U937 was sourced from European collection of cell cultures. Gold nanoparticles $30 \mathrm{~nm}$ and $80 \mathrm{~nm}$ diameter were purchased from BBI solutions. CellTiter 96®Aqueous One was purchased from Promega. Absolute ethanol, phosphate buffer saline tablets (one tablet dissolved in $200 \mathrm{~mL}$ DI water yields $0.01 \mathrm{M}$ phosphate buffer, pH 7.4) were purchased from Fisher Scientific. Thereafter, the use of 'PBS' refers to 0.01 M PBS, pH 7.4.

\section{Method}

Synthetic procedures. Silica nanoparticles were synthesised according to well established reverse-microemulsion method. ${ }^{17}$ This procedure yields highly monodisperse spherical nanoparticles and enables effective encapsulation of fluorescent dyes. ${ }^{7}$

Dye precursor formation. In a dried glass vial fluorescent dye FITC $(2.5 \mathrm{mg})$ was dissolved in $2 \mathrm{~mL}$ of 1-hexanol and APTMS 
$(5.6 \mu \mathrm{L})$ was added. The reaction was allowed to proceed over 2 hours under nitrogen atmosphere. After that $0.666 \mathrm{~mL}$ of mixture was collected and added into prepared microemulsion.

Formation of microemulsion. All nanoparticles were formed in a microemulsion prepared by combining cyclohexane $(7.5 \mathrm{~mL})$, 1-hexanol (1.133 mL), Triton ${ }^{\circledR}$ X-100 (1.894 g), DI water (0.48 $\mathrm{mL}$ ) in a $30 \mathrm{~mL}$ plastic bottle under constant stirring.

Synthesis of NP-FITC. Firstly, the microemulsion was prepared as described above. Into this $100 \mu \mathrm{L}$ of TEOS was added followed by $0.666 \mathrm{~mL}$ dye precursor solution. After $30 \mathrm{~min} 40 \mu \mathrm{L}$ of ammonium hydroxide was added to trigger polymerisation. The mixture was stirred for further 24 hours. After that the microemulsion was broken by adding $30 \mathrm{~mL}$ ethanol. Formed silica cores were purified by centrifugation (14 $000 \mathrm{rpm}, 10 \mathrm{~min}$ ) and re-dispersed in $30 \mathrm{~mL}$ ethanol $(3 \times)$. After purification, the nanoparticles were stored in ethanol solution at $4{ }^{\circ} \mathrm{C}$.

Synthesis of $\mathrm{NP}-\mathrm{A}-\mathrm{FITC}-\mathrm{NH}_{2}$. Silica nanoparticle core was synthesised similarly to NP-FITC protocol, silica nanoparticle core was synthesised similarly to NP-FITC protocol. After $24 \mathrm{~h}$ stirring two $50 \mu \mathrm{L}$ portions of TEOS were added to the mixture 30 min apart of each other. 30 min later APTMS $(25 \mu \mathrm{L})$ was added, the mixture was stirred for further 24 hours and purified as mentioned above.

Synthesis of $\mathrm{NP}-\mathrm{B}-\mathrm{FITC}-\mathrm{NH}_{2}$. Similar to the protocol above, after $24 \mathrm{~h}$ of stirring, one portion of $50 \mu \mathrm{L}$ of TEOS was added to the microemulsion. After additional $30 \mathrm{~min}$, ATPMS $(200 \mu \mathrm{L})$ was added and the mixture was stirred for further $24 \mathrm{~h}$ and purified as mentioned above.

Synthesis of $\mathrm{NP}-\mathrm{MB}-\mathrm{NH}_{2}$ and $\mathrm{NP}-\mathrm{NaFlu}-\mathrm{NH}_{2}$. Methylene blue and fluorescein sodium salt dyes were encapsulated in nanoparticle cores by using $0.48 \mathrm{~mL}$ of their aqueous solutions (6.25 $\mathrm{mM}$ ) as the water phase in the microemulsion. The rest of the procedure was similar to NP-B-FITC- $\mathrm{NH}_{2}$.

\section{Sample preparation}

Preparation of nanoparticle-loaded gels. The solid form of the gelator was dissolved in filtered DI water via mechanical stirring at $30{ }^{\circ} \mathrm{C}$ to reach concentration of $2 \mathrm{mg} \mathrm{mL}^{-1}$. Meanwhile, $1 \mathrm{mg}$ of nanoparticles was isolated from the stock solution by centrifugation. The pellet was then re-dispersed in $1 \mathrm{~mL}$ of gel solution by ultra-sonication for $20 \mathrm{~s}$ using the Sonics vibra-cell probe at $20 \%$ amplitude. This sonication step secured nanoparticles' maximum monodispersity and also triggered hydrogel formation, which normally occurred in the next few minutes. The formation of self-supporting gel could be observed by naked eye and was simply confirmed by turning the vial upside down.

Preparation of nanoparticle's aqueous solutions. Aqueous solutions of nanoparticles were prepared in a similar manner to nanoparticle gel-samples omitting the use of Fmoc-Gal.

Preparation of AuNPs-loaded gels. The solution of Fmoc-Gal was prepared as mentioned above. $2 \mathrm{~mL}$ of $30 \mathrm{~nm}$ and $80 \mathrm{~nm}$ of AuNPs solutions $\left(2 \times 10^{11}\right.$ particles per $\mathrm{mL}$ and $1.1 \times 10^{10}$ particles per $\mathrm{mL}$ respectively) were centrifuged and washed several time with water in order to remove the stabilising solution in which AuNPs are normally stored. The soft pellet obtained after centrifugation was finally redispersed in the Fmoc-Gal solution and sonicated as described above to allow the formation of the self-sustainable gel.

Preparation of AuNP's aqueous solutions. Aqueous solutions of nanoparticles were prepared in a similar manner to AuNPs gel-samples omitting the use of Fmoc-Gal.

\section{Characterisation}

Dynamic light scattering. DLS experiments were performed using Malvern Zetasizer. All nanoparticles' dispersions in DI water, PBS $0.01 \mathrm{M}$ and ethanol were typically analysed at concentrations $0.5,1.0$ and $2.0 \mathrm{mg} \mathrm{mL}^{-1}$. In the case of the AuNPs, DLS analysis was carried on $10 \times$ diluted samples.

Transmission electron microscopy. TEM micrographs were obtained using a Joel JEM-3200FS transmission electron microscope operated at $150 \mathrm{kV}$. Sample preparation involved carefully pipetting the solution of the NP-gel and NP-solution samples in water (approximately $5 \mu \mathrm{L}$ ) onto 'Carbon Films on 400 Mesh Grids Copper’ from Agar Scientific.

Fluorescence and absorbance analysis. All measurements were performed on Tecan Infinite M200 Pro Safire microplate reader using standard, Nunc Maxisorb 96-well plates. The excitation/emission wavelengths $471 / 525 \mathrm{~nm}$ were used for NPFITC, NP-A-FITC-NH $\mathrm{N}_{2}$ and NP-B-FITC-NH $\mathrm{N}_{2}$. 456/518 nm were used for NP-NaFlu- $\mathrm{NH}_{2}$. The absorbance of methylene blue of NP-MB-NH $\mathrm{N}_{2}$ was detected at $664 \mathrm{~nm}$. Tecan Infinite M200 Pro was also used for the MTS assay. The absorbance of the MTS reagent was measured at $490 \mathrm{~nm}$.

Nanoparticles tracking analysis (NTA). All measurements were performed on NanoSight LM10-HS instrument. Videos were recorded with a CCD camera and processed using Nanoparticles Tracking Analysis software. Only AuNPs samples have been analysed with this techniques and the samples were diluted two times before the analysis.

\section{Analysis}

Processing of nanoparticle-gels and nanoparticle-solutions. Prior the TEM, DLS analysis, the nanoparticle samples were stored in gels for a specific period of time. The gel was then 'broken' by agitation or vortex till the sample turned into liquid. The sample was diluted with water reaching concentration $0.5 \mathrm{mg} \mathrm{mL}{ }^{-1}$ NPs. In some cases, different concentration of NPs solution has been prepared by further dilutions. Solution-stored nanoparticle samples were processed in the same way to the gel samples.

Analysis of the dye-leaching of gel-/solution-stored nanoparticles. SiNP-gel sample and SiNP-solution sample were prepared following the procedure described above using DI filtered water and PBS $0.01 \mathrm{M}$ as media. After 28 days of storage at $8{ }^{\circ} \mathrm{C}$ both samples were processed as described above obtaining two solutions - SiNPs from gel-storage and solutionstorage. Both solutions were centrifuged (10 min, $14000 \mathrm{rpm})$, $600 \mu \mathrm{L}$ of the supernatant from the top-part of the tube was collected and the amount of dye was quantified. This was done to avoid contamination by any un-centrifuged nanoparticles. The remaining supernatant was discarded; the pellet of isolated 
SiNPs was re-dispersed in equal amount of solvent ( $2 \mathrm{~mL}$ ). 200 $\mu \mathrm{L}$ of each solution were pipetted into 96-well plate and fluorescence and absorbance values were recorded.

Analysis of the dye-leaching of NPs when stored in different solvents. Similarly to previous method, $1 \mathrm{mg}$ of NPs was redispersed in $2 \mathrm{~mL}$ of ethanol, water and PBS $0.01 \mathrm{M}$ respectively. The samples were then stored at $8{ }^{\circ} \mathrm{C}$ and the dyeleeching was observed after 1, 2, 7, 14 and 21 days following the previous protocol. Centrifuged pellets were always redispersed into the same solvent (ethanol, water, or PBS 0.01 $\mathrm{M})$ and fluorescence/absorbance measurements were taken.

Alternatively $1 \mathrm{mg}$ of NPs was suspended into $2 \mathrm{~mL}$ of water, or ethanol and shaken at $37^{\circ} \mathrm{C}$. The signal of the dye leached from the NPs to the solvent was detected every 2 hours for 8 hours, following the same procedure mentioned above.

Cytotoxicity study. MTS assay was carried on using U937 cell line (human hematopoietic cell line from histiocytic lymphoma). The cells were cultured in RPMI medium supplemented with $1 \%$ FBS and antibiotics. The cytotoxicity was analysed for three samples. NP-in-gel and NP-in-solution were prepared following the protocol mentioned above using filtered water as solvent. The third sample analysed was the interaction of cells with gel but without nanoparticles. The gel was prepared by solubilizing $2 \mathrm{mg}$ of gelator in $1 \mathrm{~mL}$ of filtered water in the absence of the nanoparticles. After sonication the gel was formed.

All three samples were shaken by hand (to break the gel) and vortexed for $1 \mathrm{~min}$ at $500 \mathrm{rpm}$. They were then diluted up to by factor of 4 , using the cell culture medium (RPMI).

The cells were plated at a density of $3 \times 10^{3}$ per well in a 96-well plate. In each well the cells were treated with $20 \mu \mathrm{L}$ of each samples, reaching a concentration of $50 \mu \mathrm{g}$ of NPs per well. The total volume of $100 \mu \mathrm{L}$ per well was reached by adding the cell culture medium. All plates were incubated at $37{ }^{\circ} \mathrm{C}$ with $5 \% \mathrm{CO}_{2}$ for up to 4 hours. After $1 \mathrm{~h}, 2 \mathrm{~h}$ and $4 \mathrm{~h}, 20 \mu \mathrm{L}$ of MTS solution was added to the each well. The plate was then incubated for further 2 hours to allow MTS reagent to react. The absorbance was read at $490 \mathrm{~nm}$ to determine the cell viability. For the calculation of cell viability, a calibration curve was done measuring the absorbance of different cell-concentrations after 2 hours incubation with MTS.

\section{Acknowledgements}

G. Giovannini, F. Kunc and C. C. Piras are thankful to Medway School of Pharmacy, University of Kent Faculty of Sciences Research fund for providing funding for their postgraduate studies.

\section{References}

1 D. Pooja, H. Kulhari, D. J. Adams and R. Sistla, Expert Opin. Ther. Pat., 2016, 26, 745-749.

2 A. Costa, M. Pinheiro, J. Magalhaes, R. Ribeiro, V. Seabra, S. Reis and B. Sarmento, Adv. Drug Delivery Rev., 2016, 102, 102-115.

3 K. Yoncheva, B. Tzankov, M. Popova, V. Petrova and N. Lambov, J. Dispersion Sci. Technol., 2016, 37, 113-118.

4 K. Petersson, D. Ilver, C. Johansson and A. Krozer, Anal. Chim. Acta, 2006, 573, 138-146.
5 R. P. Bagwe, L. R. Hilliard and W. H. Tan, Langmuir, 2006, 22, 4357-4362.

6 T. L. Moore, L. Rodriguez-Lorenzo, V. Hirsch, S. Balog, D. Urban, C. Jud, B. Rothen-Rutishauser, M. Lattuada and A. Petri-Fink, Chem. Soc. Rev., 2015, 44, 6287-6305.

7 E. J. W. Verwey, J. Phys. Colloid Chem., 1947, 51, 631-636.

8 B. V. Derjaguin, Y. I. Rabinovich and N. V. Churaev, Nature, 1978, 272, 313-318.

9 H. Kamiya and M. Iijima, Sci. Technol. Adv. Mater., 2010, 11, 044304.

10 M. Sapra, A. A. Pawar and C. Venkataraman, J. Colloid Interface Sci., 2016, 464, 167-174.

11 C. K. Kim, P. Ghosh and V. M. Rotello, Nanoscale, 2009, 1, 61-67. 12 A. Das, R. Chadha, N. Maiti and S. Kapoor, J. Nanopart., 2014, 2014, 7.

13 Y. Zhao, Z. Wang, W. Zhang and X. Jiang, Nanoscale, 2010, 2, 2114-2119.

14 R. I. Nooney, E. McCormack and C. McDonagh, Anal. Bioanal. Chem., 2012, 404, 2807-2818.

15 A. Burns, H. Ow and U. Wiesner, Chem. Soc. Rev., 2006, 35, 1028-1042.

16 L. S. de Lara, V. A. Rigo and C. R. Miranda, Eur. Phys. J. B, 2015, 88, 261-271.

17 Y. S. Lin, N. Abadeer and C. L. Haynes, Chem. Commun., 2011, 47, 532-534.

18 C. Fang, N. Bhattarai, C. Sun and M. Zhang, Small, 2009, 5, 1637-1641.

19 S. W. Bae, W. Tan and J.-I. Hong, Chem. Commun., 2012, 48, 2270-2282.

20 M. X. Yu and J. Zheng, ACS Nano, 2015, 9, 6655-6674.

21 J. L. Provis and D. G. Vlachos, J. Phys. Chem. B, 2006, 110, 3098-3108.

22 C. J. Moore, H. Monton, R. O'Kennedy, D. E. Williams, C. Nogues, C. Crean and V. Gubala, J. Mater. Chem. B, 2015, 3, 2043-2055.

23 P. Thoniyot, M. J. Tan, A. A. Karim, D. J. Young and X. J. Loh, Adv. Sci., 2015, 2, 13.

24 J. Wang, S. Banerji, N. Menegazzo, W. Peng, Q. Zou and K. S. Booksh, Talanta, 2011, 86, 133-141.

25 E. Marsich, A. Travan, I. Donati, A. Di Luca, M. Benincasa, M. Crosera and S. Paoletti, Colloids Surf., B, 2011, 83, 331-339.

26 G. S. Alvarez, C. Helary, A. M. Mebert, X. L. Wang, T. Coradin and M. F. Desimone, J. Mater. Chem. B, 2014, 2, 4660-4670.

27 C. P. Silveira, L. M. Apolinário, W. J. Fávaro, A. J. Paula and N. Durán, ACS Biomater. Sci. Eng., 2016, 2, 1190-1199.

28 S. R. Sershen, S. L. Westcott, N. J. Halas and J. L. West, J. Biomed. Mater. Res., 2000, 51, 293-298.

29 X. Hu, X. Hao, Y. Wu, J. Zhang, X. Zhang, P. C. Wang, G. Zou and X.-J. Liang, J. Mater. Chem. B, 2013, 1, 1109-1118.

30 Y. Liu, H. Meng, S. Konst, R. Sarmiento, R. Rajachar and B. P. Lee, ACS Appl. Mater. Interfaces, 2014, 6, 16982-16992.

31 H. Wu, G. Yu, L. Pan, N. Liu, M. T. McDowell, Z. Bao and Y. Cui, Nat. Commun., 2013, 4, 1943.

32 M. Zhou, A. M. Smith, A. K. Das, N. W. Hodson, R. F. Collins, R. V. Ulijn and J. E. Gough, Biomaterials, 2009, 30, 2523-2530.

33 R. P. Bagwe, C. Yang, L. R. Hilliard and W. Tan, Langmuir, 2004, 20, 8336-8342. 